\title{
A preliminary study of the efficacy
} of the polyherbal preparation Sao Thong Tai for erectile dysfunction among elderly men: a double-blind, randomized controlled trial

\author{
Chakariya Lin ${ }^{1}$, Junya Pattraraachachai ${ }^{1}$, Kammal K. Pawa ${ }^{1}$ and Surapote Wongyai ${ }^{2 *}$ (1)
}

\begin{abstract}
Background: The traditional Thai polyherbal formulation "Sao Thong Tai" (STT) contains four medicinal plants, namely Boesenbergia rotunda (L.) Mansf., Sida acuta Burm.f., Dactyloctenium aegyptium (L.) Willd., and Oryza sativa L. and it is considered an aphrodisiac and remedy for the effective treatment of decreased male libido in Thai traditional medicine and it may ameliorate erectile function. This pilot study was carried out to investigate the efficacy and safety of the polyherbal STT for mild to moderate erectile dysfunction in a small group of elderly men in Thailand in a doubleblind, randomized controlled trial.

Methods: This research is a preliminary study. Fifty-four elderly males aged 50-69 years who had been diagnosed with mild to moderate ED were randomized into two groups of equal size: an STT group $(n=27)$ and a placebo control group $(n=27)$. The patients received either an $800 \mathrm{mg}$ dose of STT extract or a lactose placebo twice a day for the eight-week treatment period, after which they were followed up for four weeks. Erectile function, sexual activity, selfreported attitudes toward sexual dysfunction, and partner-reported sexual dysfunction were investigated to confirm the efficacy of the polyherbal preparation. The International Index of Erectile Function (IIEF-5) and the Scale for Quality of Sexual Function (QSF) were used for primary outcome assessment.
\end{abstract}

Results: The IIEF-5 scores of the STT group were significantly increased compared with those of the placebo group $(p<0.001)$. The severity of ED decreased from "mild" or "mild to moderate" to normal in $88.9 \%$ of the STT group vs. $0 \%$ of the placebo group, and the QSF score increased in the STT group $(p<0.001)$. Sexual activity, self-reported views of sexual dysfunction, and partners' views of sexual dysfunction showed significant improvement in the STT group, and there was no significant difference in psychosomatic quality of life. No adverse events were observed in either group.

Conclusion: Compared with a placebo, an oral aqueous extract of STT taken daily at a dose of 1,600 mg for eight weeks can effectively treat mild to moderate ED in elderly men. The preparation was also shown to be safe with respect to all parameters assessed. This finding demonstrates that STT can be used for the effective treatment of decreased male libido in Thai traditional medicine and may ameliorate ED.

Trial registration: TCTR/20180126001 033/2560. Registered 16 January 2018, Thai Clinical Trials Registry http://www. thaiclinicaltrials.org/

Keywords: Sao Thong Tai, Polyherbal Thai traditional preparation, Erectile dysfunction, Efficacy, Safety

\footnotetext{
*Correspondence: surapote@rsu.ac.th

${ }^{2}$ College of Oriental Medicine, Rangsit University, 52/347 Phaholyothin

Rd, Lak Hok, Mueang Pathumtani District, Pathumtani 12000, Thailand

Full list of author information is available at the end of the article
} 


\section{Introduction}

Sexual health is an essential aspect of one's quality of life and it is an essential parameter correlated with human wellbeing. Erectile dysfunction (ED) in males leads to dissatisfaction with their sex life and deterioration of their sexual well-being [1]. The Massachusetts Male Aging Study report indicated that, in a communitybased sample, $52 \%$ of noninstitutionalized men aged 40-70 years experienced ED [2-4]. In addition, the same study revealed that the prevalence of ED was positively associated with age [4]. Men in their seventies and eighties had the highest prevalence of ED [5]. As a result of cultural differences between Asian and Western countries, it has been reported that most Asian men with ED prefer to seek their own treatment [2]. Furthermore, it is common for them to seek traditional herbal medicine as a potential option. It has been estimated that over 152 million men worldwide will suffer from ED by 2025 [6]. Similarly, in Thailand, the expected prevalence rate of ED will be $42.18 \%$ of the male population by that date [7]. As the population ages, safe and effective interventions that preserve male sexual function are needed. ED treatment has rapidly evolved, and new knowledge has continued to expand the options for treatment since 1960. Currently, there are twelve treatments available, each classified as internal or external. The most popular internal treatments relying on oral pharmacotherapy are PDE5 inhibitors and soluble guanylate cyclase activators. In comparison, the external treatments commonly used are penile supporters, vacuum devices, and endovascular tools [8].

More recent studies have confirmed the effectiveness of pharmacological and nonpharmacological treatments, including natural products and herbal medicines, in treating ED. In systematic reviews on ED treatments, pharmacological interventions or combined therapies were found to be more effective than nonpharmacological interventions [9]. Pharmacological intervention (including PDE5 inhibitors, penile injections, and testosterone) was effective in treating ED. However, there are limitations associated with these interventions related to responsiveness, adherence and side effects. A recent review of medicinal plants in the regulation and management of male ED demonstrated that diverse medicinal plant species and bioactive active compounds are preferred as therapeutic options because they are natural, abundant, readily available, and inexpensive resources with few or no side effects [10]. Many herbs traditionally used as aphrodisiacs have shown as a potential source of PDE5 inhibitors [11]. They have been demonstrated effective in vivo and in vitro for the mitigation and treatment of ED. The activity of promising medicinal plants for the treatment of male ED is due to their potential healing effects. Top medicinal plants, including Curcuma longa, Myrica rubra, Pausinystalia johimbe, Lepidium meyenii, and Panax ginseng, are traditionally used as aphrodisiacs and treatments for ED [10, 12]. In addition, a recent systematic review on alternative medicine and herbal remedies in the treatment of ED concluded that there is promising evidence in support of the herbs Panax ginseng, pycnogenol, Prelox and Tribulus terrestris and the nutritional supplement L-arginine as ED treatments based on International Index of Erectile Function (IIEF) scores [13]. Furthermore, nanotechnology has been used to develop products for ED treatment, such as nanoparticles loaded with nitric oxide, sialorphin, sonic hedgehog or herbal medicine and nanoparticle-associated adipose tissue-derived stem cells, which are potential new treatments for ED [14]. Although medicinal plants and herbal medicines have demonstrated their potential as sources of medicine for ED, further clinical studies are warranted to further assess the evidence supporting them. Patients' physical condition and the severity of their ED also affect the success of treatment. Some limitations in their effectiveness are inevitable. The risk factors for male ED are diabetes, hypertension, obesity, lack of physical activity, alcoholism, cigarette smoking, use of certain drugs, and psychological factors [9]. Treating ED with herbal medicine has become an increasingly popular alternative option for many patients worldwide, since medicines such as traditional Chinese medicine (TCM), Indian traditional medicine, and Thai traditional medicine (TTM) have been used since ancient times by local people. Some of the best traditional herbal treatments have survived for thousands of years and have been gradually modified or improved over time. The long-term survival and local popularity of the use of these traditional plants are evidence of their relative effectiveness and safety, as verified by thousands of healing practitioners.

Based on a total of 280 relevant articles reviewed by the authors, it was found that 105 medicinal plants with aphrodisiac potential have long been used in treating ED, such as Pausinystalia johimbe (K.Schum.) Pierre ex Beille in Africa [15], Eurycoma longifolia Jack in Malaysia [16], Crocus sativus L. in Iran [17], and Boesenbergia rotunda (L.) Mansf. in Thailand [18]. Several herbs are used as active ingredients in a traditional Thai preparation called Sao Thong Tai (STT), which has been used as an aphrodisiac remedy for the effective treatment of decreased male libido in TTM pharmacopeia since ancient times [19], and it may ameliorate ED. It also has diuretic properties, promotes kidney health, and strengthens the body [20]. This herbal medicinal preparation is an equal combination of four medicinal plants (Boesenbergia rotunda (L.) Mansf., Sida acuta Burm.f., Dactyloctenium aegyptium (L.) Willd., and Oryza sativa L.), and it is prepared 
by decoction with boiling water [19]. The main active ingredient is of $B$. rotunda and it's major chemical constituents are flavonoids, which may inhibit PDE5 [18, 21 ] and promote sexual arousal [18]. One flavone in $B$. rotunda is 5,7-dimethoxyflavone which has been proven to be a promising PDE5 inhibitor [22]. D. aegyptium is a diuretic and nourishes the kidneys, while the other two herbs strengthen the body [20]. Clinical trials to support and demonstrate the traditional use of STT and potential therapeutics to ameliorate ED are limited. Therefore, their therapeutic efficacy, safety and tolerability for ED can be investigated only by randomized, controlled trials. Accordingly, this pilot clinical study aimed to provide scientific evidence supporting STT as an aphrodisiac and remedy for the effective treatment of decreased male libido in TTM, as well as its amelioration of ED. The research was carried out to verify the efficacy and safety of the preparation for mild and moderate ED among elderly men in Thailand in a double-blind, randomized controlled trial, and the main measures of success were cure rate, pleasure and partner satisfaction. Erectile function, sexual activity, self-reported views of sexual dysfunction, and partners' views of sexual dysfunction were investigated to confirm the efficacy of this herbal preparation.

\section{Materials and Methods Chemical and reagents}

High-performance liquid chromatography (HPLC)-grade methanol and acetonitrile were purchased from J.T. Baker (Phillipsburge, USA). Analytical-grade glacial acetic acid was purchased from Merck (Darmstadt, Germany). HPLC-grade water was obtained from RCI Labscan (Bangkok, Thailand). Standard cardamonin, pinocembrin and pinostrobin were purchased from Sigma (St. Louis, USA). Pharmaceutical-grade lactose monohydrate was obtained from DFE Pharma GmbH \& Co. KG (Goch, Germany). All other reagents were of analytical grade unless stated otherwise.

\section{Preparation of STT capsules}

The herbs used in this study consisted of an aqueous dry extract (drug-to-extract ratio: 3.3:1 w/w; extraction solvent: water; excipients: none) of an STT formulation prepared from equal parts (25\% each) of dry plant materials, including the rhizome of $B$. rotunda, the whole plant of $S$. acuta, the whole plant of $D$. aegyptium, and the seed of $O$. sativa. The starting raw materials were obtained from Charoensuk Osod Co., Ltd. (Nakon Pathom, Thailand). Each component was verified by comparing the HPLC-profile [23] against authenticated reference plant materials identified by Assoc. Prof. Nitsiri Ruangrungsi, a botanist, and deposited at the College of Public Health
Sciences, Chulalongkorn University, Thailand, with identifier codes BR0332017, SA0332017, DA0332017, and OA0332017. The fresh herbs were dried at $60{ }^{\circ} \mathrm{C}$ and then reduced to a powder, after which each botanical was identified and assayed for the content of the respective marker compounds using HPLC [23]. The powdered dry botanical raw materials were mixed in equal proportions to obtain a homogeneous mixture, which was then decocted in boiling water with a drug to solvent ratio of 1:10. The aqueous extract was spray dried into a free-flowing powder without carriers or other excipients at a drug-to-extract ratio of 3.3:1. The dose of the STT extract, $800 \mathrm{mg}$, was calibrated to contain the same quantities of marker compounds present in one dose of the traditional formulation prepared by decoction using boiling water [19]. Four hundred milligrams of the powdered dry extract was loaded into each capsule. The quantities of the marker constituents per capsule were as follows: $0.28 \mathrm{mg}$ pinocembrin, $0.29 \mathrm{mg}$ cardamonin and 0.43 mg pinostrobin, as determined by a modified RP-HPLC method [24]. The placebo group received capsules with the same color and labeling, filled with $400 \mathrm{mg}$ of lactose powder.

\section{Quality control of STT capsules by HPLC}

RP-HPLC was used as an analytical tool to identify characteristic peaks representing each active ingredient in STT capsules and determine the quantity of each marker compound by using a modified RP-HPLC method [24]. Chromatography was performed on an HPLC apparatus (Thermo Scientific, CA, USA) equipped with a Spectra System pump P4000, a Spectra System autosampler AS3000, and a Spectra System UV6000LP diode array detector. Separation was performed on a VDSpher PUR 100 C18-E column $(250 \times 4.6$ mm i.d., $5 \mu \mathrm{m})$ (Chromatographie Technik GmbH, Berlin, Germany). The elution was carried out with gradient delivery systems with a flow rate of $1.0 \mathrm{~mL} \mathrm{~min}^{-1}$ at ambient temperature. The mobile phase consisted of $2 \%(\mathrm{v} / \mathrm{v})$ acetic acid (solvent A) and acetonitrile (solvent B). The total running time was $50 \mathrm{~min}$, and the linear gradient program was as follows: from 0 to $20 \%$ B in $10 \mathrm{~min}, 20 \%$ B for $5 \mathrm{~min}$, to $60 \%$ $\mathrm{B}$ in $15 \mathrm{~min}$, to $80 \% \mathrm{~B}$ in $10 \mathrm{~min}$, to $100 \% \mathrm{~B}$ in $10 \mathrm{~min}$, and $100 \% \mathrm{~B}$ for $10 \mathrm{~min}$; flow rate $1 \mathrm{~mL} \mathrm{~min}^{-1}$; detection: UV $254 \mathrm{~nm}$; and injection volume $20 \mu \mathrm{l}$. The column was washed with acetonitrile for $10 \mathrm{~min}$ after each analysis and equilibrated with $2 \%(\mathrm{v} / \mathrm{v})$ acetic acid for $10 \mathrm{~min}$ before each injection. UV detection was set at a wavelength of $254 \mathrm{~nm}$. Chromatograms were recorded and processed with ChromQuest 4.2.34 version 3.1.6 data acquisition system (ThermoFinigan, San Jose, CA, USA) chromatographic software. 


\section{Acute oral toxicity study of STT capsules}

The preparation was investigated in vivo in acute toxicity studies and included two experiments. The first acute oral toxicity test in animals was conducted in mice according to Test Guideline (TG) No.420: Acute Oral Toxicity-Fixed Dose Method (Limit test) of the OECD Guidelines for Testing of Chemicals (2001) [25] with dosing 2,000 mg/kg body weight. The second acute oral toxicity test in animals was conducted in Wistar rats according to Test Guideline (TG) No.423: Acute Oral Toxicity-Acute Toxic Class Method of the OECD Guidelines for Testing of Chemicals (2001) [26] with two doses (2,000 and 5,000 $\mathrm{mg} / \mathrm{kg}$ body weight). The overall observation period of both studies was 14 days. In-depth toxicity tests demonstrated the safety of the formulation in both experiments [27].

\section{Clinical study}

This research design was a comparison between two groups, the treatment and placebo groups. The research was carried out at a traditional Thai medicine outpatient clinic in Bangkok, Thailand. Urologists and general physicians took responsibility for the participants for an eight-week treatment period and a subsequent four-week follow-up, with the patients participating at various times from March 2017 to December 2017. The study was performed in accordance with the Guideline for Good Clinical Practice (ICH-6) and Declaration of Helsinki. The trial received approval from the Human Research Ethics Committee of Thammasat University, Thailand (reference number MTU-EC-OO-0-188/59). It was also registered in the Thai Clinical Trials Registry with approval ID TCTR/20180126001 033/2560.

\section{Participants}

Inclusion criteria The inclusion criteria were as follows. After signing an informed consent form, married men aged 50-69 years old who had stable chronic medical illnesses, such as controlled diabetes mellitus and/or hypertension, and had suffered from ED symptoms for at least six months were enrolled in the study. Each participant had also been in a stable heterosexual relationship for at least six months; both partners agreed to attempt intercourse at least once a week on average during the study, and men with bioavailable testosterone $>1.50 \mathrm{ng} /$ $\mathrm{dL}$ and IIEF-5 scores of 11-21 (mild to moderate ED) were eligible for inclusion.

Exclusion criteria Those with the following conditions were excluded: 1) a history of major hematological, renal or hepatic disorders; stroke; myocardial infarction; or cardiovascular disease within the last six months; 2) current peptic ulcers or bleeding disorders; 3) a history of prostate cancer; 4) elevated prostate-specific antigen (PSA $\geq 4 \mathrm{ng} / \mathrm{mL}$ ); 5) major uncontrolled psychiatric disorder; 6) penile anatomical abnormalities; 7) primary hypoactive sexual desire; 8) history of alcohol or drug abuse 9) resting hypotension (resting systolic blood pressure $90 \mathrm{mmHg}$ or diastolic pressure $50 \mathrm{mmHg}$ ); 10) resting hypertension (resting systolic blood pressure 170 $\mathrm{mmHg}$ or diastolic pressure $110 \mathrm{mmHg}$ ); 12) body mass index BMI over $30 \mathrm{~kg} / \mathrm{m}^{2}$; 13) use of herbal products or drugs containing testosterone or any androgenic activity in the month before the start of the trial; 14) abnormal levels of thyroid-stimulating hormone (TSH), T3, or T4; and 15) history of allergy to herbal products. In addition, participants who were unable to take at least $80 \%$ of the scheduled treatment dose were removed from the trial. Participants had the right to withdraw from the study for any reason at any time.

\section{Intervention}

The participants were randomized to either the treatment or placebo group, as indicated above. The treatment group received a dose of two STT capsules (400 mg per capsule) twice a day before breakfast and dinner for up to 8 weeks and was followed up for 4 weeks after treatment. The placebo group consumed lactose capsules at the same dose as the treatment group. The pharmacist managed the preparation and delivery of the medicines according to the patients' random group assignments.

\section{Outcomes}

Primary outcomes The efficacy of STT compared with placebo was assessed using IIEF-5 scores [28] in the Thai version validated for use in Thailand [29]. This sexual health inventory for men classifies ED severity as follows: 1-7 severe ED, 8-11 moderate ED, 12-16 mild to moderate ED, 17-21 mild ED and 22-25 no ED. The patients used this self-assessment instrument to monitor and report their status during the 12-week follow-up period of the study. The five essential domains of the IIEF are erectile function, intercourse satisfaction, orgasmic function, sexual desire, and overall satisfaction. Furthermore, we evaluated sexual function quality using the Scale for Quality of Sexual Function (QSF) [30]. Thirty-two items representing four dimensions were used to measure sexual function; three of the dimensions (psychosomatic score, sexual activity and self-reported sexual function) were based on the patients' own reports, and the fourth 
dimension was the viewpoint of the partner regarding sexual function.

Secondary outcomes Total serum testosterone levels were assessed at baseline and at the four- and eight-week follow-ups. During the follow-up period, adverse drug reactions and adverse events in general were monitored and recorded. Adverse events were classified into four levels of severity (none, mild, moderate, or severe), and their association with the drug used in the study was classified into three levels (probably, unlikely, or unknown). A medical examination including laboratory blood chemistry tests and urinalysis was performed at baseline and at the time of each follow-up visit. All blood and urine tests were analyzed at the Professional Laboratory Management Corp Co., Ltd. (Bangkok, Thailand), an accredited laboratory, in compliace with ISO 15189, (4005/46).

\section{Sample size}

The data for the analysis were collected at four visits (on the starting date and at four-week intervals thereafter), and repeated-measures ANOVA was selected in advance as the statistical method; the necessary sample size was calculated using the $G^{*}$ Power program based on the planned design and statistical analysis. Based on a systematic review capturing the variation in the effect of herbal preparations on the mean difference in IIEF- 5 [31], the lower bound of the medium effect size (0.31) was chosen as the effect size for the sample size calculation [32]. The calculation was based on repeatedmeasures ANOVA with four timepoints. A statistical significance threshold (1- $\alpha)$ of $95 \%$ and a power $(1-\beta)$ of $80 \%$ were used. Equal sample sizes (1:1) were used for the treatment and placebo groups. G*Power sample size calculation software was applied to compute the number of required subjects [33]. The calculated minimum number of subjects per group was 27 , with a total necessary sample size of 54 subjects.

\section{Randomization and blinding}

A urologist screened a total of 62 participants. Fifty-four patients satisfied the criteria and were randomized to either the treatment group $(n=27)$ or the placebo control group $(n=27)$. During the study, no participant was terminated (Fig. 1). The baseline characteristics and severity of sexual dysfunction were comparable (Table 1).

The study groups were created using simple randomization. The allocation of the cases to the two groups was determined by randomly drawing papers inscribed with codes by a third person, who had no conflict of interest regarding the research. The code letter "A" referred to the group taking herbal medicine, while the code letter "B" referred to the control group taking placebo capsules. The random assignments were double-blinded, with neither the physician nor the participants knowing which subjects belonged to each group.

\section{Statistical analysis}

We generated a table with a frequency comparison, along with the $p$-value from the chi-square or Fisher's exact test. Student's $t$ test was used to compare means between the groups at baseline. Statistical analysis was conducted using repeated-measures ANOVA for the primary outcomes. Follow-up means were compared within groups, and the mean difference between groups at each visit was considered a measure of the effectiveness of STT. The means of the primary outcomes were estimated using the generalized linear model (GLM) adjusted for the covariates. Furthermore, the GLM was used to estimate relative change. A statistical significance threshold of $5 \%$ was used for all of the tests.

\section{Results}

\section{Quality control of STT capsules by HPLC}

The HPLC chromatogram of STT capsules, with identification data for 11 well-resolved peaks representing 4 active ingredients, is shown in Fig. 2. The components and their peaks are as follows: D. aegyptium (peaks 1-3), S. acuta (peak 4), B. rotunda (peaks 5-7, 9-11), and O. sativa (peak 8). The quantities of 3 known marker compounds from B. rotunda, namely, pinocembrin (peak 7), cardamonin (peak 9) and pinostrobin (peak 10), were $0.28,0.29$ and $0.43 \mathrm{mg}$ per capsule, respectively.

\section{Acute oral toxicity study of STT capsules}

The results of the oral acute toxicity study of STT capsules investigated in vivo in mice and rats over a period of 14 days showed the safety of the formulation in both experiments [27]. During the observation period all mice and rats survived and there was no sign of toxicity. All mice and rats appeared normal and gained weight and no mortality occurred. Necropsy findings at termination did not show any significant gross pathological lesions.

\section{Clinical study}

Of a total of 62 participants screened by the urologist, a total of 54 patients satisfied the criteria and were randomized to either the treatment group $(n=27)$ or placebo group $(\mathrm{n}=27)$. During the study, no participant was terminated (Fig. 1). The baseline characteristics and severity of sexual dysfunction were comparable between the two groups (Table 1). 


\section{Enrollment}

Assessed for eligibility $(n=62)$

Excluded $(n=8)$

- Not meeting inclusion criteria $(n=8)$

- Declined to participate $(n=0)$

- Other reasons $(n=0)$

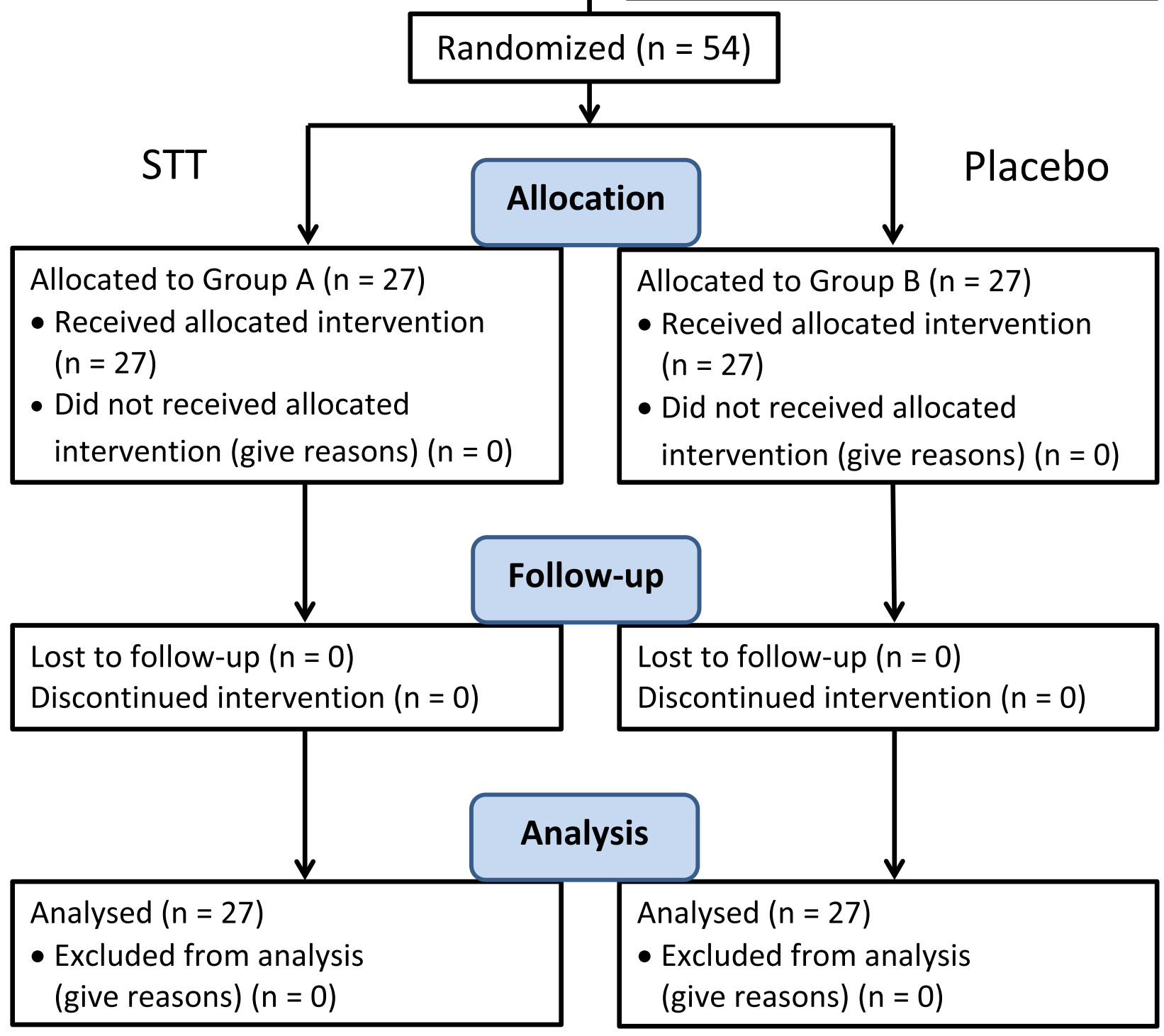

Fig. 1 Consolidated Standards of Reporting Trials (CONSORT) flow chart

Baseline characteristics

Regarding the baseline characteristics of the two groups (Table 1 ), the obesity and IIEF-5 scores were similar. The participants were classified as normal or overweight/ obese according to their BMI, with a cutoff value of $25 \mathrm{~kg} / \mathrm{m}^{2}$. The mean body mass values of the STT and 


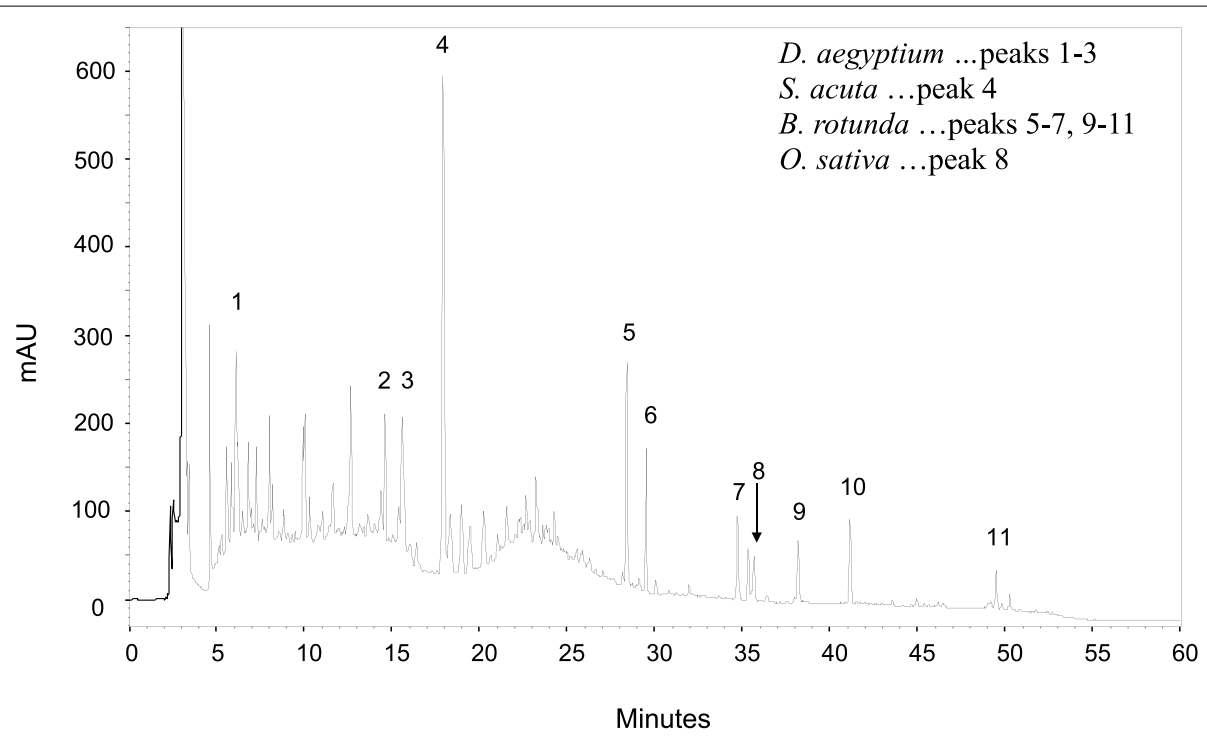

Fig. 2 Chromatogram of the contents of Sao Thong Tai (STT) capsules with characteristic peaks representing the active ingredients: peaks 1-3) D. aegyptium; peak 4) S. acuta; peaks 5-7, 9-11) B. rotunda; and peak 8) O. sativa. Each capsule contained the following markers: peak 7, pinocembrin $0.28 \mathrm{mg}$; peak 9, cardamonin $0.29 \mathrm{mg}$; and peak 10) pinostrobin $0.43 \mathrm{mg}$. The chromatographic conditions were characterized as follows: stationary phase, VDSpher PUR 100 C18-E, $250 \times 4.6$ mm i.d., 5 m; mobile phase, A: 2\% (v/v) acetic acid in water; mobile phase, B: acetonitrile; gradient: 0 to $20 \%$ B in 10 min, 20\% B for 5 min, to 60\% B in 15 min, to 80\% B in 10 min, to 100\% B in 10 min, 100\% B for 10 min; flow-rate $1 \mathrm{~mL}$ min-1; detection, $254 \mathrm{~nm}$; and injection volume $20 \mu \mathrm{L}$

Table 1 Mean and standard error of the mean (SEM) of participants' characteristics at baseline

\begin{tabular}{llll}
\hline Characteristics & $\begin{array}{l}\text { STT }(\boldsymbol{n}=\mathbf{2 7}) \\
\text { mean } \pm \text { SEM }\end{array}$ & $\begin{array}{l}\text { Placebo }(\boldsymbol{n}=\mathbf{2 7}) \\
\text { mean } \pm \text { SEM }\end{array}$ & $\boldsymbol{p}$-value \\
\hline Age (yrs.) & $54.6 \pm 0.9$ & $58.6 \pm 1.1$ & 0.009 \\
Marriage (yrs.) & $22.0 \pm 1.8$ & $27.5 \pm 1.5$ & 0.022 \\
Weight (kg) & $74.0 \pm 2.2$ & $68.4 \pm 2.0$ & 0.067 \\
Waist (in) & $34.9 \pm 0.6$ & $34.3 \pm 0.7$ & 0.558 \\
BMl (kg/m²) & $26.0 \pm 0.7$ & $25.3 \pm 0.7$ & 0.463 \\
IEF-5 & $16.3 \pm 0.5$ & $16.7 \pm 0.4$ & 0.052 \\
\hline
\end{tabular}

placebo groups were 74.0 and 68.4 kilograms, respectively, which fell within the overweight category. However, the BMI and waist measurements of both groups were in the normal range. The mean \pm standard error values of the IIEF- 5 scores were $16.37 \pm 0.57$ in the STT group and $16.70 \pm 0.42$ in the placebo group. The mean age of the placebo group ( $58.6 \pm 1.1$ yrs.) was significantly greater than that of the STT group $(54.6 \pm 0.9$ yrs.) $(p=0.009)$. The average length of marriage also differed significantly between the STT and placebo groups $(p=0.022)$. Table 2 reports the results of the eight-week laboratory tests. Samples with high glycated hemoglobin (HbA1c) and fasting plasma glucose (FPG; uncontrolled blood sugar) levels constituted similar proportions of the STT and placebo groups from the beginning to the end of the treatment. Hypertension status followed a similar pattern during follow-up.

\section{Efficacy Primary outcomes}

IIEF-5 Scores A total of 54 patients (27 STT and 27 placebo) were included in the study. Compared with the placebo group, the STT group showed a significant increase in IIEF-5 scores from the second visit (week $4)$ to the fourth visit (week 12) (Fig. 3a) $(p<0.001)$. The means and $p$-value shown in the figure were estimated using GLMs after controlling for the covariates (age and years of marriage). The mean total IIEF scores in the STT group increased from 16.5 during the first visit (day 0) to 22.7 during the fourth visit (week 12), while the mean for the placebo group remained stable at approximately 16. The mean scores of the STT group began to show a significant difference at the second visit (week 4), and the difference between the treatment and placebo groups remained significant throughout the study. Based on responses to the questionnaire items in the erectile function domain, including confidence, hardness, frequency and satisfaction, 15/27 (55.6\%) of the subjects in the STT group were within the score range of "mild" (score values of 17-21) at the first visit (day 0). At the end of the study, $88.9 \%$ of the STT group reached the score range of "nor$\mathrm{mal} / \mathrm{no}$ ED" (score values of 22-25). For item Q.2 "Over 
Table 2 Medical health during the period of treatment (8 weeks)

\begin{tabular}{lllll}
\hline Laboratory tests & Timepoint & $\begin{array}{l}\text { STT } \\
\mathbf{n = 2 7}\end{array}$ & $\begin{array}{l}\text { Placebo } \\
\mathbf{n = 2 7}\end{array}$ & $\mathbf{p}^{@}$ \\
\hline HbA1c > 6.5 & $1^{\text {st }}$ (Day 0) & 7 & 10 & 0.379 \\
& $2^{\text {nd }}$ (Week 4) & 8 & 12 & 0.260 \\
FPG > 126 & $3^{\text {rd }}$ (Week 8) & 8 & 11 & 0.393 \\
& $1^{\text {st }}$ (Day 0) & 2 & 3 & 1.000 \\
& $2^{\text {nd }}($ Week 4) & 2 & 2 & 1.000 \\
Hypertension & $3^{\text {rd }}$ (Week 8) & 3 & 3 & 1.000 \\
& $1^{\text {st }}$ (Day 0) & 11 & 7 & 0.248 \\
& $2^{\text {nd }}$ (Week 4) & 10 & 6 & 0.233 \\
& $3^{\text {rd }}$ (Week 8) & 8 & 6 & 0.535 \\
\hline
\end{tabular}

@ Chi-squared test/Fisher's exact test the past 6 months, when you had erection with sexual stimulation, was your erection hard enough for penetration?", 24 of 27 patients in the STT group were within the range of "no ED" (22-25) at the end of the study (Fig. 3b). In the STT group, $55.6 \%$ of the subjects had "mild" ED at the first visit (day 0), which decreased to $7.4 \%$ during the fourth visit (week 12); the rate of "mild to moderate" ED decreased to $3.7 \%$, and no "moderate to severe" ED existed. The placebo group showed no cases of recovery to the "normal/no ED" level before and after the end of the study, but some changes were observed; specifically, the rate of "mild" cases decreased from $63.0 \%$ to $48.2 \%$, and the rate of "mild to moderate" cases increased from $37.0 \%$ to $52.8 \%$.

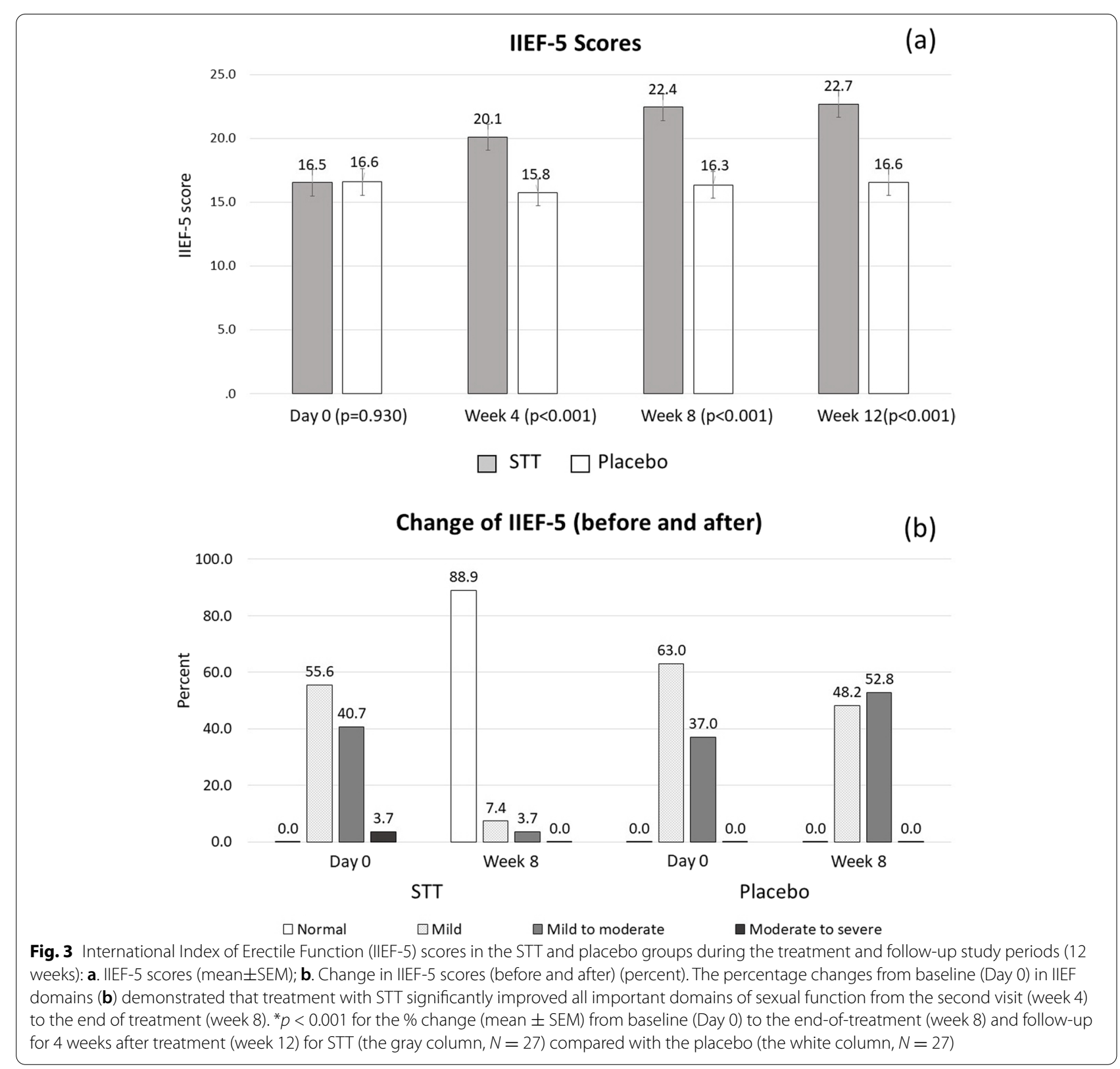




\section{Mean QSF scores}

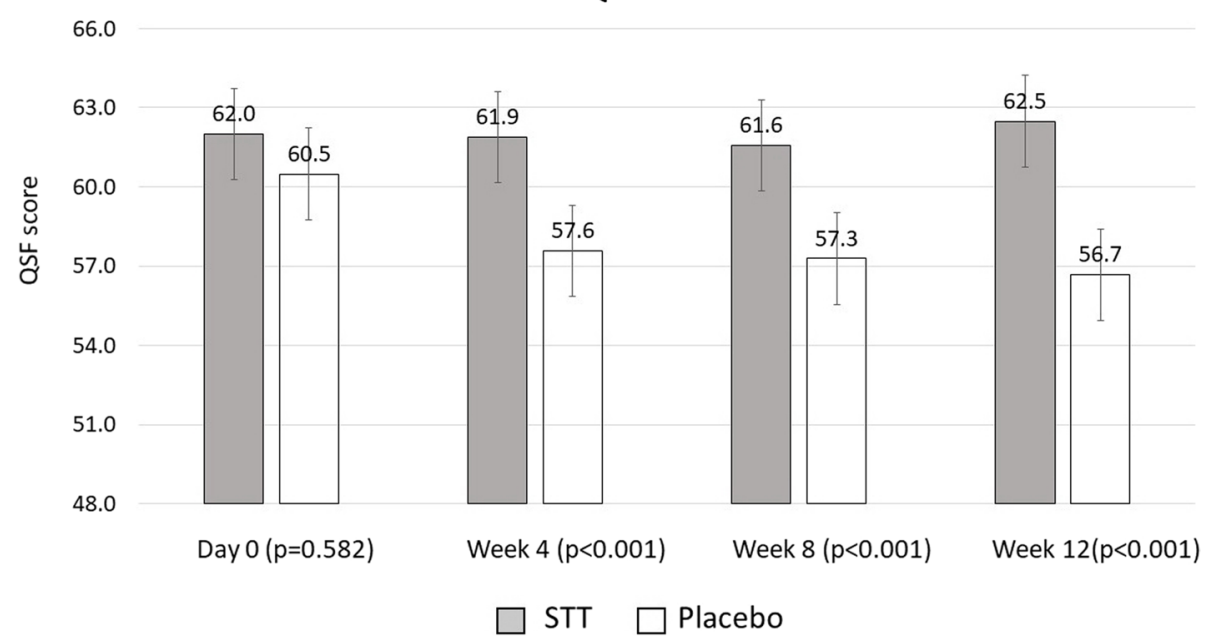

Fig. 4 The mean Quality of Sexual Function (QSF) scores between the STT and placebo groups during the study period: The mean QSF scores showed a significant increase in the STT group (the gray column, $N=27)(p<0.001)$ from the second visit (week 4$)$ to the end of treatment (the fourth visit, week 12), whereas the scores of the placebo group (the white column, $N=27$ ) decreased gradually to the end of the follow-up period

QSF Scores The mean QSF scores exhibited differences from the second visit (week 4) to the fourth visit (week 12) (Fig.4). The QSF scores showed a significant increase in the STT group $(p<0.001)$, whereas the scores of the placebo group decreased gradually to the end of the follow-up (Fig. 4). The changes in each dimension of the QSF (Fig. 5a-d) [psychosomatic quality of life (Fig. 5a), sexual activity (Fig. 5b), self-reported sexual function (Fig. 5c), and the partner's view of sexual function (Fig. 5d)] were similar to those in the total QSF scores. Regarding the overall sexual activity (Q.14-22) level scores, the treatment group began to exhibit significantly different scores $(p<0.001)$ from the second visit $(20.1 \%)$ to the fourth visit $(25.7 \%)$, with the degree of intensity/severity ranging from "Slightly" to "Strongly". Sexual self-reports began to show differentiation during the third visit (week 8$)(p<0.043)$, and the partners' views of sexual function became differentiated during the last visit (week 12) $(p=0.002)$. There was no significant difference in psychosomatic quality of life (Fig. 5a) between the treatment and placebo groups. However, the personal situation regarding Q.113 (psycho-somatic quality of life) (Fig. 5a) in the treatment group itself improved from visit 2 (week 4) (21.6) to visit 4 (week 12) (16.0), indicating that the severity of the symptoms decreased.

\section{Secondary outcome}

According to the standard laboratory tests performed in the treatment and placebo groups at baseline and during the follow-up period, no clinically significant changes were noted in most of the laboratory parameters throughout the study. These tests included blood urea nitrogen $(\mathrm{BUN})$, serum creatinine $(\mathrm{Cr})$, alanine transaminase (ALT), aspartate transaminase (AST), alkaline phosphatase (ALP), uric acid, prostate-specific antigen (PSA), thyroid stimulating hormone (TSH), triiodothyronine (T3) and thyroxine (T4). The values were in the reference ranges. However, the blood sugar levels were slightly higher than the reference ranges in both groups because the participants were elderly and some had stable chronic medical illnesses.

Regarding testosterone levels, including total serum testosterone, there were no significant differences between the STT and placebo groups before, during, or after treatment, and bioavailable testosterone was found to be in the reference range. The mean scores of bioavailable testosterone from the first visit to the third visit declined in both groups, and there were no significant differences between groups $(p=0.12, p=0.14$, and $p=0.24)$ (Table 3).

\section{Safety}

No adverse events caused by treatment were recorded in either the STT group or the placebo group. Six participants had ankle pain, Achilles tendinitis, and muscle pain, due to physical exercise. Five participants had slight eye discomfort at the beginning of the drug intake period, and the two participants who reported lumbar pain had been suffering from moderate pain before enrollment. 


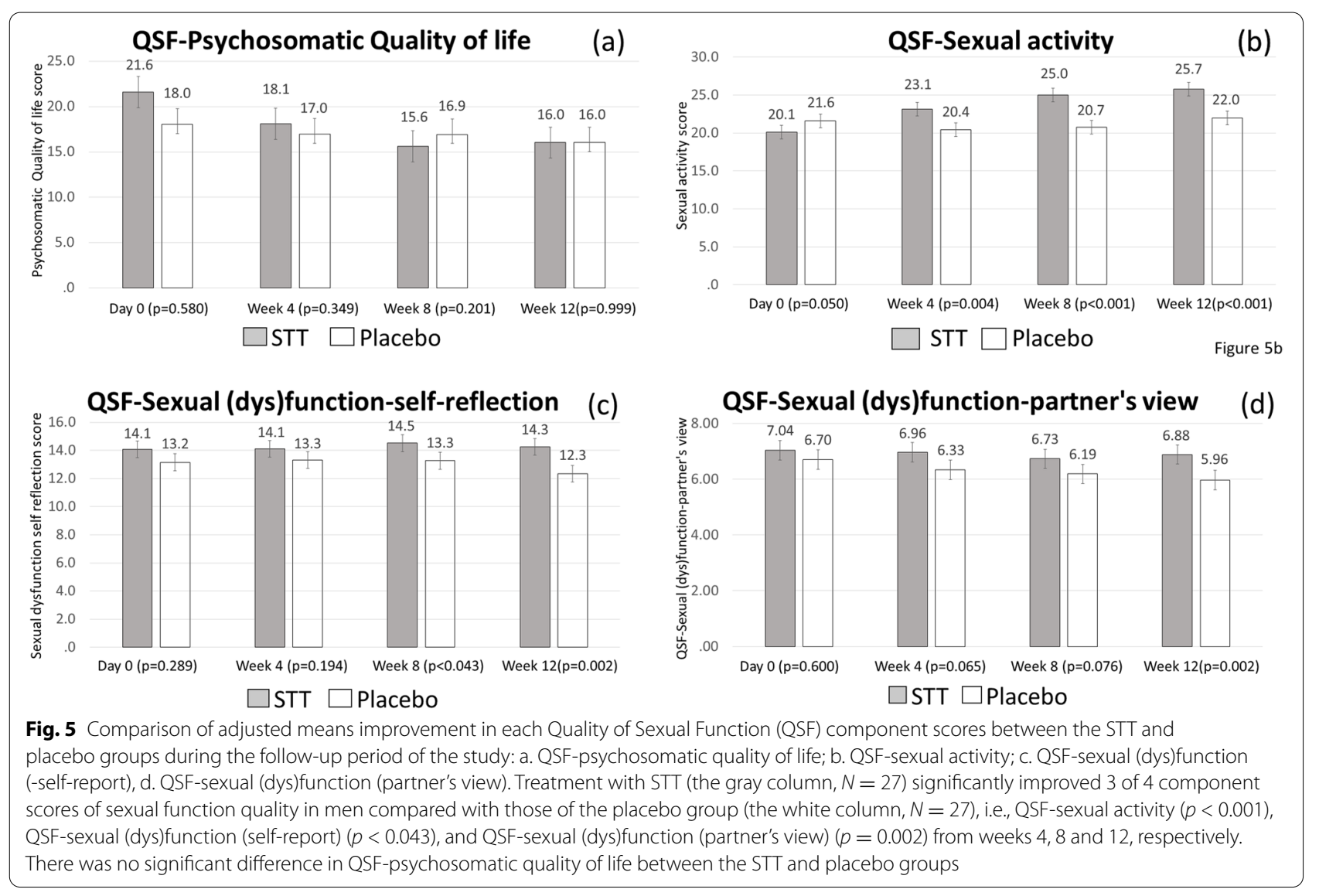

\section{Discussion}

\section{Quality control of STT capsules by HPLC}

The chromatographic profiles of STT capsules (Fig. 2) could be used for analysis of this herbal medicine by controlling the amounts of 3 known markers, pinocembrin (peak 7), cardamonin (peak 9) and pinostrobin (peak $10)$, from $B$. rotunda and evaluating the ratios between the characteristic peaks representing the active ingredients and known markers. However, it is still necessary to identify all characteristic peaks representing the other 3 active ingredients to improve the rigor of this quality control method. The present study provides a useful basis for the development of quality control procedures for this herbal medicine by using characteristic compounds or chemical markers.

\section{Acute oral toxicity study of STT capsules}

The treated mice and rats did not exhibit STT toxicity. STT extract was recognized as safe and non toxic.

\section{Clinical study}

STT is a well-known traditional herbal preparation in Thailand. In addition to its traditional use as a remedy for the effective treatment of decreased male libido, which may ameliorate erectile function, data from randomized controlled clinical trials on its efficacy and safety are lacking. The present clinical trial in 54 male patients with mild to moderate ED aged 50-69 years old for 12 weeks who received an aqueous extract of STT 1,600 mg/day or placebo was conducted as a result. This study emphasized treatment response, in terms of drug efficacy and tolerability (side effects), and treatment satisfaction, in terms of subjective patient and partner responses and overall satisfaction with treatment based on self-report questionnaires.

The five domains in the IIEF- 5 questionnaire are erectile function, orgasmic function, sexual desire, intercourse satisfaction, and overall satisfaction. Total IIEF-5 scores can be classified into five levels of severity, where a higher score represents better function; the score ranges are $5-7,8-11,12-16,17-21$ and $22-25$ for severe, moderate to severe, mild to moderate, mild, and no ED, respectively. The score range used to recruit subjects for the study (8-21) was centered around mild to moderate $[28,29]$. The results of the IIEF-5 (Fig. 3a-b) showed that the degree of erection from initial sexual stimulation to the completion of intercourse was sufficient for penetration. The firmness increased steadily over the 
Table 3 Mean, standard error of the mean (SEM), and p-value for the effect of treatment on testosterone levels between the STT and placebo groups

\begin{tabular}{|c|c|c|c|c|c|}
\hline \multirow[t]{2}{*}{ Laboratory tests } & \multicolumn{2}{|l|}{ STT } & \multicolumn{2}{|c|}{ Placebo } & \multirow[t]{2}{*}{$p$-value } \\
\hline & Mean & SEM & Mean & SEM & \\
\hline $1^{\text {st }}$ visit testosterone $(\mathrm{SI}: \mathrm{nmol} / \mathrm{L})$ & 20.40 & 1.88 & 18.14 & 1.60 & 0.36 \\
\hline $2^{\text {nd }}$ visit testosterone (SI: $\left.\mathrm{nmol} / \mathrm{L}\right)$ & 20.08 & 1.76 & 18.36 & 1.71 & 0.48 \\
\hline $3^{\text {rd }}$ visit testosterone (SI: $\left.\mathrm{nmol} / \mathrm{L}\right)$ & 19.93 & 1.78 & 17.61 & 1.57 & 0.33 \\
\hline $1^{\text {st }}$ visit testosterone (conventional: $\mathrm{ng} / \mathrm{mL}$ ) & 5.89 & 0.54 & 5.23 & 0.46 & 0.36 \\
\hline $2^{\text {nd }}$ visit testosterone (conventional: $\mathrm{ng} / \mathrm{mL}$ ) & 5.63 & 0.50 & 5.35 & 0.49 & 0.68 \\
\hline $3^{\text {rd }}$ visit testosterone (conventional: $\mathrm{ng} / \mathrm{mL}$ ) & 5.75 & 0.51 & 5.04 & 0.47 & 0.31 \\
\hline $1^{\text {st }}$ visit bioavailable testosterone (calculated) & 1.71 & 0.08 & 1.94 & 0.12 & 0.12 \\
\hline $2^{\text {nd }}$ visit bioavailable testosterone (calculated) & 1.75 & 0.08 & 1.99 & 0.13 & 0.14 \\
\hline $3^{\text {rd }}$ visit bioavailable testosterone (calculated) & 1.69 & 0.09 & 1.91 & 0.15 & 0.24 \\
\hline
\end{tabular}

follow-up period, and as a result, 24 out of 27 patients in the treatment group improved from mild, mild to moderate, or moderate to severe and had normal scores by the fourth visit (week 12), corresponding to an overall cure rate of $88.9 \%$; no such improvement was observed in the placebo group. The primary outcome was the average IIEF-5 score, which showed a significant mean difference between groups. After the mean difference was calculated, the proportion or percent of recovery change for each severity level was considered. The statistical analysis took age into account when estimating means using GLMs and performing significance tests. Controlling for the effect of age explains why the recovery rate in the placebo group was different from the value reported in previous research. A comparison of the efficacy, using IIEF-5 scores, of STT in this study $(\mathrm{N}=54)$ with that of Tribulus terrestris $(N=180)$ [34] and Korean Red Ginseng $(\mathrm{N}=60)$ [35] for the treatment of mild to moderate ED in male patients demonstrated cure rates of 88.9, 100.0 and $66.6 \%$, respectively. STT demonstrated potential effectiveness for the treatment of mild to moderate ED compared with that of the two herbs.

The QSF scale consists of psychosomatic quality of life, sexual activity, self-reported sexual (dys) function, and sexual (dys) function in the partners' view [30]. The study results for psychosomatic quality of life showed that the patients' scores decreased from the second visit (week 4) (18.1) to the fourth visit (week 12) (16.0) in the treatment group (Fig. 5a), especially in terms of sleep problems. After treatment, the degree of sleep quality of 16 of the 27 patients improved from "moderate to no improvement". After the fourth visit, the overall scores for psychosomatic quality of life showed no difference between the treatment and placebo groups (16.0). The scores for sexual activities such as a regular sex life and desire for sexual activity (sexual intercourse or masturbation) in the QSF group increased steadily from the second visit (23.1) to the fourth visit (25.7) compared with those of the placebo group (22.0) (Fig. 5b). Meanwhile, the QSF scores reflecting desire for sexual intercourse doubled in 20 patients compared with the baseline values, and 23 patients reported masturbation. Based on the score for self-reported sexual (dys) function, including sexual dreams, fantasies, or desire, as reported by the patients and their partners, there was no difference between the groups before or after treatment (Fig. 5c). Fig. $5 d$ shows the partner-reported sexual (dys) function scores, including sexual organ response, sexual excitement, and satisfaction during sexual activities. The results showed no difference from the second visit (7.0) to the fourth visit (6.9) in the treatment group. Similar results were also found in the placebo group. However, 18 of the 27 patients in the treatment group achieved high satisfaction during sexual activity (Question 32). Based on the improvement displayed in all five domains of the IIEF-5 (Fig. 3a-b) and the significant increase in the QSF (Fig. 4, Fig. 5a-d), the herbal extract STT helped to enhance the overall quality of sexual behavior in elderly men and the sexual satisfaction of both the patients and their partners.

Colson's report indicates that the incidence of ED increases with age [36]. Older adult men try to find treatment either from modern medicine or traditional and alternative medicine. Recently, many research studies on ED treatment using conventional therapy and complementary and alternative medicine have been conducted both locally and globally. Ower's study is one of several studies on the use of TTM for ED treatment; these drugs, prepared from raw materials, have been locally used for hundreds of years. Thai herbal preparations, including STT, commonly consist of various ingredients that mitigate side effects. The primary compounds in the drug used by the treatment group are alkaloids, saponins, and 
flavonoids, which are bioactive aphrodisiac compounds. Through the action of these compounds, STT is capable of affecting penile erection [37]. The word "aphrodisiac" is from the Greek name "Aphrodite," which is associated with sex, love, and beauty [38]; accordingly, one of the primary uses of an aphrodisiac drug is to promote sexual desire. The process begins from the central nervous system, directly controlling blood flow to the male penis [37]. The process occurs whenever there is a source of sexual stimulation such as visual fantasies, erotic thought, masturbation, or coitus. These factors stimulate the parasympathetic nerves to secrete and release nitric oxide from the smooth muscle cells of the cavernous artery. Moreover, the enzyme guanylate cyclase (GC) is stimulated to convert guanosine triphosphate (GTP) into cyclic guanosine triphosphate (cGMP). This process results in relaxation of the smooth muscle of the penis, thereby dilating the penile arteries, which causes increased blood circulation and erection of the penis. The erection of the penis can be stopped by the enzymatic hydrolysis of cGMP to GMP by phosphodiesterase type 5 (PDE5) inside the tissue. Therefore, aphrodisiacs inhibit the hydrolysis of cGMP to extend the time of erection $[8,36,38]$. Kotta classified aphrodisiacs into three main categories based on their mechanisms [39]. The first category provides nutritional value to improve health or well-being, leading to improved sexual performance and libido. The second exerts physiological effects on blood flow to numb the genital area during sexual activity. The third category crosses the blood-brain barrier and stimulates brain areas related to sexual arousal.

In this study, the STT herbal extract improved sexual performance by promoting sexual arousal. In particular, B. rotunda (Krachai in Thai) is a selective inhibitor of PDEs, especially PDE5, which supports the properties of this preparation [40]. PDE5 is the main isoenzyme of PDE associated with cGMP; its metabolic action relaxes the vessels and increases the flow of blood to the corpora cavernosa, resulting in erectile function during sexual intercourse. A recent review reported that several herbal medicines can affect penile erection through different mechanisms [37]. The ergogenic properties of alkaloids lead to dilation of the blood vessels, causing an erection. Additionally, saponin reacts to form nitrous oxide and leads to smooth muscle relaxation via the L-arginine/ nitrous oxide pathway [37]. Finally, the presence of flavonoids in plant extracts can influence androgen levels.

In a review of scientific studies, Ongwisespaiboon and Jiraungkoorskul presented evidence that phytochemical substances or secondary plant metabolites have sexual arousal properties and can be classified into three main groups of aphrodisiacs based on their similar structures [18]. These categories are (1) flavonoids and other phenolics, (2) alkaloids, and (3) saponins. Functionally, they can act as PDE inhibitors, particularly sildenafil, used clinically to treat male ED. The chemical compounds of the herbal preparation in STT were alpinetin, boesenbergin, cardamonin, geraniol, krachaizin, panduratin $\mathrm{A}$, pinostrobin, pinocembrin, rotundaflavone, and silybin [18] in B. rotunda; alkaloids, saponins and flavonoids in S. acuta; and saponins, flavonoids, and alkaloids in D. aegyptium. During our 12-week study, STT enabled patients to achieve sexual arousal; in this manner, STT improved sexual behavior, including sexual desire, sexual motivation, sexual performance, and sexual pleasure. O. sativa is used in Thai folk medicine as a tonic, aphrodisiac, and diuretic as well as to nourish the kidneys and spleen [20]. Protein, B-complex vitamins, and minerals in rice help to build resilient muscles, nourish skin and blood vessels, and support the endocrine system. Furthermore, rice provides iron to increase the hematocrit; phosphorus and potassium to maintain internal water balance; and other nutrients with various functions. Thus, rice helps to restore homeostasis.

Our study also addressed the quality of sleep and quality of life by employing within-patient repeated measures for the Pittsburgh Sleep Quality Index (PSQI) and ShortForm 36 quality of life instrument (SF-36). Improvements in sleep quality were found from the second visit to the fourth visit in the QSF, PSQI, and SF-36. The total mean SF-36 scores increased steadily from the first visit (120.0) to the fourth visit (128.5) in the treatment group, while those of the placebo group were 126.1 at the first visit and 125.3 at the fourth visit. The results showed that STT improved sleep quality compared with the placebo, indicating that this herbal preparation has a beneficial effect on sleep and leads to sleep improvement because of sexual satisfaction. Testosterone levels did not significantly differ among the first, second, and third visits in either group (Table 3). Thus, STT showed no effect on testosterone levels and the same results as those in the randomized control trials of Tribulus terrestris [34] and Korean Red Ginseng [35] for the treatment of mild to moderate ED in male patients.

The safety of STT in terms of blood chemistry was assessed by comparing ALT, AST, ALP, uric acid, thyroid function (T3, T4, TSH), and PSA in the periods before and after the treatment. There were no statistically significant changes in either group. The traditionally used water extracts of STTs are described as being safe, which is supported by the present trial showing no differences in all analyzed safety parameters and adverse events compared with the placebo. Negative consequences associated with treatment have been identified as important to patients receiving treatment for ED [41]. There was no statistically significant difference in adverse event 
rates between the groups, and no serious adverse events were reported. Ankle, lumbar, and muscle pain was found in both groups due to physical training. Minor eye discomfort was reported during the first two days. One patient in the placebo group reported discomfort in the form of a slight headache because the patient was recovering from a cold at the time. Furthermore, eight out of 11 patients who had hypertension after taking STT at the first visit (day 0) returned to normal by the third visit (week 8) because STT may promote vasodilation, thereby decreasing the resistance to blood flow.

The participant groups were followed up during the trial using a careful tracking system, and excellent relationships were maintained with the participants. The researcher made periodic calls to the participants, provided advice and counseling during the study and made appointments, which were recorded in writing at all times. All 54 participants completed the full trial and brought back the medicine bottles at each follow-up visit; the researchers recorded the number of doses remaining at each follow-up to confirm that the capsules were taken as scheduled.

STT provides an effective and low-cost alternative ED treatment that has several advantages over other options, which are more expensive and have other adverse effects. The traditional treatments of lifestyle modifications, oral medications, injections, and penile prostheses fail to provide the desired results for a large proportion of patients suffering from ED [6]. Novel approaches using low-intensity extracorporeal shock wave therapy (Li-ESWT), stem cell therapy (SCT), and platelet-rich plasma (PRP) to treat ED still need further investigation [14]. Oral PDE5 inhibitors (i.e., sildenafil, tadalafil, and vardenafil) take effect within 1 hour and are active for up to 24 hours (tadalafil); however, a key disadvantage of these drugs is that they are contraindicated in men with severe cardiovascular disease and men on nitrates [42, 43]. Additionally, PDE5 inhibitors are expensive for a first-line treatment, costing over $\$ 10$ per dose [42]. TTM is also highly effective, has no known life-threatening side effects, acts as a targeted therapy, is inexpensive, and strengthens the body while treating ED; however, patients must avoid seafood and fermented food during treatment [44]. This research supports the traditional use of STT in elderly men for improving the quality of sexual function and demonstrates the potential to be used as an alternative therapy in patients with mild to moderate ED.

\section{Limitations and future studies}

The first limitation of this study is that it was a preliminary study using only a small group of patients to investigate the efficacy of STT for the treatment of mild to moderate ED; further research should repeat these tests in a larger sample. It would also be of interest to further study the efficacy of STT in patients with moderate to severe ED. Second, the age range of the participants should be expanded to 40-70 years to recruit a more representative sample of ED patients. Third, the IIEF-5 mainly investigates erectile function, and the efficacy of the product with respect to other functions requires further investigation. Finally, since the PSQI demonstrated that the quality of sleep in the treatment group improved remarkably over that of the control group, STT has been shown to be effective in terms of sleep quality. Due to the positive effects of STT in treating ED, further investigations are needed to determine whether this preparation can also accelerate ED treatment in various populations that were not represented in the current study, including patients with the following conditions: renal disorders, alcohol or drug abuse, resting hypotension (resting systolic blood pressure $90 \mathrm{mmHg}$ or diastolic pressure $50 \mathrm{mmHg}$ ), resting hypertension (resting systolic blood pressure $170 \mathrm{mmHg}$ or diastolic pressure $110 \mathrm{mmHg}$ ), BMI over $30 \mathrm{~kg} / \mathrm{m}^{2}$, and the use of herbal products or drugs that contain testosterone or have any androgenic activity. Therefore, further research in this area is warranted.

\section{Conclusion}

In this preliminary study a double-blind, randomized controlled trial in 54 male patients with mild to moderate ED demonstrated significant improvements in libido, sexual performance, satisfaction, and physical functioning resulting from aqueous STT, a Thai traditional herbal medicine consisting of $B$. rotunda, $S$. acuta, $D$. aegyptium and $O$. sativa. The preparation was potentially shown to be effective for treating mild to moderate (scores 8-21) ED in elderly men without causing serious side effects. The daily dose of $1,600 \mathrm{mg}$ of STT extract for 8 weeks is well tolerated and safe compared with the placebo. This finding demonstrates that STT can be used as an effective aphrodisiac treatment of decreased male libido in TTM and can ameliorate ED. However, more evidence with a larger number of patients and further studies of STT are required to further evaluate the efficacy, safety and quality of this preparation, with the goal of identifying an effective and safe natural remedy for ED.

\section{Supplementary Information}

The online version contains supplementary material available at https://doi. org/10.1186/s40816-022-00341-4.

Additional file 1.

Additional file 2. 


\begin{abstract}
Acknowledgments
The study was supported by the Human Research Ethics Committee of Thammasat University (MTU-EC-OO-0-188/59), Assoc. Prof. Kanvee Viwatpanich, Dr.rer.nat, Assist. Prof. Aungkana Krajarng, Ph.D. at Thammasat University, Dr. Teanchai Kerasawangporn, and all of the volunteers who participated in this study. We thank Assoc. Prof. Nitsiri Ruangrungsi for providing medicinal plant authentication.
\end{abstract}

\section{Authors' contributions}

$\mathrm{CL}$, JP, KKP, and SW contributed to the protocol, study development, statistical analyses and manuscript writing. CL, KKP and SW participated in recruiting, treating and follow-up patients and registering study data. All authors revised and approved the final manuscript.

\section{Funding}

This research received a supporting grant from the Agricultural Research Development Agency (Public Organization), Bangkok, Thailand [Grant number CRP6005020490, 2017].

\section{Availability of data and materials}

All data and material sets in this study are available from the corresponding author upon request.

\section{Declarations}

\section{Ethics approval and consent to participate}

The trial received approval from the Human Research Ethics Committee of Thammasat University, Thailand (reference number MTU-ECOO-0-188/59) on 3 March 2017. It was additionally registered at the Thai Clinical Trials Registry with approval ID TCTR/20180126001 033/2560 on 16 January 2018.

\section{Consent for publication}

Not applicable.

\section{Competing interests}

None.

\section{Author details}

${ }^{1}$ Chulabhorn International College of Medicine, Thammasat University, Pathumtani 12120, Thailand. ${ }^{2}$ College of Oriental Medicine, Rangsit University, 52/347 Phaholyothin Rd, Lak Hok, Mueang Pathumtani District, Pathumtani 12000, Thailand.

Received: 12 May 2021 Accepted: 6 February 2022

Published online: 21 February 2022

\section{References}

1. Tirado LCE, Ferrer JE, Herrera AM. Aging and erectile dysfunction. Sex Med Rev. 2016;4:63-73.

2. Feldman HA, Goldstein I, Hatzichristou DG, Krane J, Mckinlay JB. Impotence and its medical and psychosocial correlates: Results of the Massachusetts Male Aging Study. J Urol. 1994;151(1):54-61.

3. Johannes CB, Araujo AB, Feldman HA, Derby CA, Kleinman KP, Mckinlay JB. Incidence of erectile dysfunction in men 40 to 69 years old: Longitudinal results from the Massachusetts Male Aging Study. J Urol. 2000;163:460-3.

4. Mobley DF, Khera M, Baum N. Recent advances in the treatment of erectile dysfunction. Postgrad Med J. 2017;93:679-85.

5. Ang HH, Ngai TH, Tan TH. Effects of Eurycoma longifolia Jack on sexual qualities in middle aged male rats. Phytomedicine. 2003;10:(6-7):590-3.

6. Kongkanand A. Thai Erectile Dysfunction Epidemiological Study Group. Prevalence of erectile dysfunction in Thailand. Int J Androl. 2000;23(Suppl 2):77-80.

7. Lewis RW, Fugl-Meyer KS, Bosch R, Fugl-Meyer AR, Laumann EO, Lizza E, et al. Epidemiology/risk factors of sexual dysfunction. J Sex Med. 2004;1(1):35-9.
8. Ismail EA, El-Sakka Al. Innovative trends and perspectives for erectile dysfunction treatment: A systematic review. Arab J Urol. 2016;14:84-93.

9. Ciocanel O, Power K, Eriksen A. Intervention to treat erectile dysfunction and premature ejaculation: An overview of systematic reviews. Sex Med. 2019;7:251-69.

10. Sin VJ-E, Anand GS, Koh H-L. Botanical medicine and natural products used for erectile dysfunction. Sex. Med Rev. 2020. https://doi.org/10. 1016/j.sxmr.2020.10.005.

11. Ganapathy AA, Priya VMH, Kumaran A. Medicinal plants as a potential source of Phosphodiesterase-5 inhibitors: A review. J Ethnopharmacol. 2021; https://doi.org/https://doi.org/10.1016/j.jep.2020.113536.

12. Masuku NP, Unuofin JO, Lebelo SL. Advances in nanoparticle delivery system for erectile dysfunction: An updated review. Sex Med. 2021. https:// doi.org/10.1016/j.esxm.2021.100420.

13. Leisegang K, Finelli. Alternative medicine and herbal remedies in the treatment of erectile dysfunction: A systematic review. Arab. J Urol. 2021;19(3):323-39.

14. Masuku NP, Unuofin JO, Lebelo SL. Promising role of medicinal plants in the regulation and management of male erectile dysfunction. Biomed Pharmacother. 2020; http://www.creativecommons.org/licenses/ by-nc-nd/4.0.

15. Ernst $\mathrm{E}$, Pittler $\mathrm{MH}$. Yohimbine for erectile dysfunction: a systematic review and meta-analysis of randomized clinical trials. J Urol. 1998;159(2):433-6.

16. Kotirum S, Ismail SB, Chaiyakunapruk N. Efficacy of Tongkat Ali (Eurycoma longifolia) on erectile function improvement: Systematic review and meta-analysis of randomized controlled trials. Complement Ther Med. 2015;23:693-8.

17. Shamsa A, Hosseinzadeh H, Molaei M, Shakeri MT, Rajabi O. Evaluation of Crocus sativus L. (saffron) on male erectile dysfunction: A pilot study. Phytomedicine. 2009;16:690-3.

18. Ongwisespaiboon $\mathrm{O}$, Jiraungkoorskul W. Fingerroot, Boesenbergia rotunda and its aphrodisiac activity. Phramacogn Rev. 2017;11:27-30.

19. Boonyachothi P. Thai traditional medicine book (Thai). Bangkok: Kasembanakij; 1970

20. The Phaetthayasatsongkhro chabap-luang, Book 3 (Wat Po) (Thai): Bangkok: Pratapjai Service Center Limited Partnership; 1986

21. Temkitthawon P, Viyoch J, Limpeanchob N, Pongamornkul W, Sirikul C, Kumpila A, et al. Screening for phosphodiesterase inhibitory activity of Thai medicinal plants. J Ethnopharmacol. 2008;119:214-7.

22. Temkitthawon P, Hinds TR, Beavo JA, Viyoch J, Suwanborirux K, Pongamornkul W, et al. Kaempferia parviflora, a plant used in traditional medicine to enhance sexual performance contains large amounts of low affinity PDE5 inhibitors. J Ethnopharmacol. 2011;137(3):1437-41.

23. Li Y, Shen Y, Yao C, Guo D. Quality assessment of herbal medicines based on chemical fingerprints combined with chemometrics approach: A review. J Pharm Biomed Anal. 2020; https://doi.org/https://doi.org/10 1016/j.jpba.2020.113215.

24. Tan BC, Tan SK, Wong SM, Ata N, Rahman NA, Khalid N. Distribution of flavonoids and cyclohexenyl chalcone derivatives in conventional propagated and in vitro-derived field-grown Boesenbergia rotunda (L.) Mansf.. J Evid Based Complementary Altern Med. 2015; http://dx.doi.org/https:// doi.org/10.1155/2015/451870.

25. Organization for Economic Co-operation and Development. OECD guideline for testing of chemicals: acute oral toxicity - fixed dose method (limit test) (OECD guideline no. 420). Adopted on 17th December 2001.

26. Organization for Economic Co-operation and Development. OECD guideline for testing of chemicals: acute oral toxicity - acute toxic class method (OECD guideline no. 423). Adopted on 17th December 2001.

27. Lin C, Pattaraarchachai J, Kumar KP, Wongyai S. Acute toxicity studies of Thai herbal preparation "Sao Thong Tai" in animals. Sci Techol Asia. 2020;25(4):106-14.

28. Rosen RC, Cappelleri JC, Gendrano N. The International Index of Erectile Function (IIEF): A state-of-the science review. Int J Impot Res. 2002:14:226-44.

29. Sangkum P, Sukying C, Viseshsindh W. Validation and reliability of a Thai version of the International Index of Erectile Dysfunction (IIEF) for Thai population. J Med Assoc Thai. 2017;100(Suppl. 9):S73-9.

30. Heinemann LAJ, Potthoff P, Heinemann K, Pauls A, Ahlers CJ, Saad F. Scale for Quality of Sexual Function (QSF) as an outcome measure for both genders? J Sex Med. 2005:2:82-95. 
31. Ciocanel O, Power K, Eriksen A. Interventions to treat erectile dysfunction and premature ejaculation: An overview of systematic reviews. Sex Med Rev. 2019;7:251-69.

32. McLeod SA. What does effect size tell you? Simply Psychology. published 2019; Available from: https://www.simplypsychology.org/effect-size.html. (accessed 10 Jul 2019).

33. Faul F, Erdfelder E, Lang AG, Buchner A. G*Power 3: A flexible statistical power analysis program for the social, behavioral, and biomedical sciences. Behav Res Methods. 2007;39(2):175-91.

34. Kamenova Z, Filevab S, Kalinovc K, Jannini EA. Evaluation of the efficacy and safety of Tribulus terrestris in malesexual dysfunction - A prospective, randomized, double-blind,placebo-controlled clinical trial. Maturitas. 2017; https://doi.org/10.1016/j.maturitas.2017.01.011.

35. Andrade E, Mesquita AA, Claro JA, Andrade PM, et al. Study of the efficacy of Korean Red Ginseng in the treatment of erectile dysfunction. Asian J Androl. 2007;9(2):241-4

36. Colson MH, Cuzin B, Faix A, Grellet L, Huyghes E. Current epidemiology of erectile dysfunction, an update. Sexologies. 2018;27:e7-13.

37. Cruz AC, Guerra NG, Souza KEBP, Eleutério IC, Silva LC, Otoni EG, et al. The action of herbal medicine on the libido: aspects of nutritional intervention in increasing sexual desire. Nutrire. 2017. https://doi.org/10.1186/ s41110-017-0051-0.

38. West E, Krychman M. Natural aphrodisiacs-A review of selected sexual enhancers. Sex Med Rev. 2015. https://doi.org/10.1002/smrj.62.

39. Kotta S, Ansari SH, Ali J. Exploring scientifically proven herbal aphrodisiacs. Pharmacogn Rev. 2013. https://doi.org/10.4103/0973-7847.112832.

40. Pham NK, Nguyen HT, Nguyen QB. A review on the ethnomedicinal uses, phytochemistry and pharmacology of plant species belonging to Kaempferia L. genus (Zingiberaceae). Pharm Sci Asia. 2021. https://doi. org/10.29090/psa.2021.01.19.070.

41. Hanson-Divers C, Jackson SE, Lue TF, Crawford SY, Rosen RC. Health outcomes variables important to patients in the treatment of erectile dysfunction. J Urol. 1998;159(5):1541-7.

42. Annual reports of Eli Lilly and Co., Pfizer, and Bayer. The PDE5 inhibitors: Viagra, Levitra, and Cialis. Available at: http://www.patienteducationcent er.org/articles/the-pde5-inhibitors-viagra-levitra-cialis/\#sthash.U9fKAjcR. dpuf. (accessed 20 Nov 2015)

43. Brock GB, McMahon CG, Chen KK, Costigan T, Shen W, Watkins V, et al. Efficacy and safety of tadalafil for the treatment of erectile dysfunction: results of integrated analyses. J Urol. 2002;168:1332-6.

44. Silajaruk Phra Chetuphon Temple (Wat Pho) Thai Pharmacopeia, Bangkok:2004: 134-43.

\section{Publisher's Note}

Springer Nature remains neutral with regard to jurisdictional claims in published maps and institutional affiliations.

\section{Submit your manuscript to a SpringerOpen ${ }^{\circ}$ journal and benefit from:}

- Convenient online submission

- Rigorous peer review

- Open access: articles freely available online

- High visibility within the field

- Retaining the copyright to your article 